\title{
Tetrabutylammonium cyanide catalyzes the addition of TMSCN to aldehydes and ketones
}

\author{
Rubén Córdoba, Aurelio G. Csákÿ, and Joaquín Plumet* \\ Departamento de Química Orgánica, Facultad de Química, Universidad Complutense, E-28040- \\ Madrid, Spain \\ E-mail:plumety@quim.ucm.es
}

Dedicated to Prof. Enrique Meléndez

(received 14 Oct 03; accepted 29 Dec 03; published on the web 11 Jan 04)

\begin{abstract}
The catalytic effect of $\mathrm{Bu}_{4} \mathrm{NCN}$ on the addition of TMSCN to the carbonyl group of spiroepoxycyclohexadienones and to some other representative carbonyl compounds has been considered.
\end{abstract}

Keywords: Ammonium salts, catalysis, trimethylsilyl cyanide, cyanohydrins

\section{Introduction}

Cyanohydrin trimethylsilyl ethers are useful synthetic intermediates for the preparation of elaborated targets. ${ }^{1}$ For this reason, a large array of catalytic species has been used for the synthesis of this kind of compounds. ${ }^{2}$ Among them, particular attention has been devoted to metal-catalyzed processes. However, the use of non-metal catalysts to carry out organic transformations is important from an environmental standpoint. In this paper we describe the results of the TMSCN addition to the carbonyl group of spiroepoxycyclohexadienones ${ }^{3}$ and certain representative carbonyl compounds catalyzed by ammonium salts.

\section{Results and Discussion}

In the context of the synthesis of cyanohydrins derived from spiroepoxycyclohexadienones, ${ }^{3}$ we have observed that the reaction of compound 1 with TMSCN (1.0 eq) in the presence of $\mathrm{Bu}_{4} \mathrm{NCN}(0.1 \mathrm{eq})$ afforded an inseparable mixture of diastereomeric cyanohydrin derivatives 2 and 3 in ratio 1:1.3 (71\% isolated yield). However, at this stage the stereochemical assignment of compounds 2 and 3 was not possible. This result contrasts with those reported by Waldmann et 
$a{ }^{4}{ }^{4}$ where the formation of bezenic derivative 4 was observed when using a molar ratio 1: TMS$\mathrm{CN}: \mathrm{Bu}_{4} \mathrm{NCN}=1: 18.4: 1$ (Scheme 1).<smiles>COC1=C(Br)C(=O)C2(C=C1Br)CO2</smiles>

1

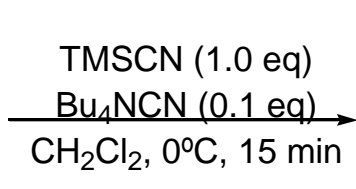

$\underset{\mathrm{CH}_{2} \mathrm{Cl}_{2}, 0^{\circ} \mathrm{C}, 15 \mathrm{~min}}{\longrightarrow}$

$\mathrm{Br}$

2

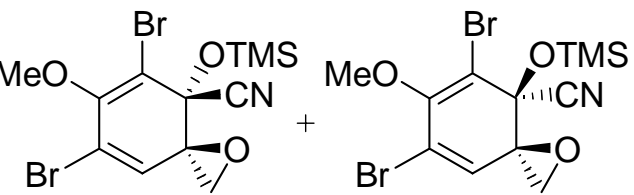

3<smiles>COc1c(Br)c(O)c(CO)c(C#N)c1Br</smiles>

4

\section{Scheme 1}

In the context of these findings, two comments should be made: i) the reaction of $\mathbf{1}$ with TMSCN in the absence of the ammonium salt resulted in the recovering of unaltered starting material; and ii) to the best of our knowledge, only an isolated report describing the use of $\mathrm{Bu}_{4} \mathrm{NCN}$ as catalytic agent for the O-TMS-cyanosilylation of 3-pentanone ( $84 \%$ isolated yield) has been previously reported. ${ }^{5}$

On the basis of these considerations, we decided to explore the scope and limitations of the use of the system $\mathrm{TMSCN} / \mathrm{Bu}_{4} \mathrm{NCN}$ (cat.) for the O-TMS-cyanosilylation of other spiroepoxycyclohexadienones and also for the same reaction using some representative carbonyl derivatives as starting materials.

The results of the OTMS-cyanosilylation of a variety of spiroepoxycyclohexadienones (Scheme 2) and some other representative carbonyl compounds (Scheme 3) are quoted in Tables 1 and 2 respectively.<smiles>[R]C1=C([R])C([R])=C([R])C2(CO2)C1=O</smiles>

5 - 7

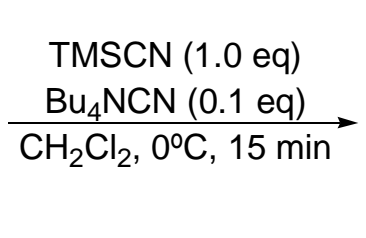

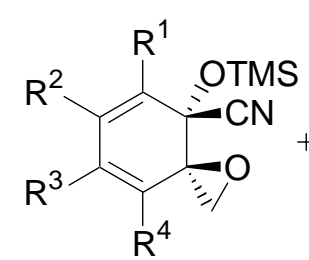

8 - 10

\section{Scheme 2}


Table 1. O-TMS cyanosilylation of spiroepoxycyclohexadienones

\begin{tabular}{cccccccc}
\hline No. & Starting material & $\mathrm{R}^{1}$ & $\mathrm{R}^{2}$ & $\mathrm{R}^{3}$ & $\mathrm{R}^{4}$ & Product (\%) & Diastereomeric ratio \\
\hline 1 & $\mathbf{5}$ & $\mathrm{Br}$ & $\mathrm{MeO}$ & $\mathrm{Br}$ & $\mathrm{MeO}$ & $\mathbf{8}(60)$ & $1: 1^{\mathrm{b}}$ \\
2 & $\mathbf{6}$ & $\mathrm{H}$ & $\mathrm{H}$ & $\mathrm{Br}$ & $\mathrm{H}$ & $\mathbf{9}(50)$ & $1: 1.4^{\mathrm{b}}$ \\
3 & $\mathbf{7}$ & $\mathrm{H}$ & $\mathrm{MeO}$ & $\mathrm{H}$ & $\mathrm{H}$ & $\mathbf{1 0}(45)$ & $1: 1.6^{\mathrm{c}}$ \\
\hline
\end{tabular}

${ }^{a}$ Isolated yield of the diastereomeric O-TMS cyanohydrins.

${ }^{b}$ Determined by GC/MS on the purified mixture of diastereomeric O-TMS cyanohydrins.

${ }^{\mathrm{c}}$ Determined by ${ }^{1} \mathrm{H}-\mathrm{NMR}$ on the purified mixture of diastereomeric O-TMS cyanohydrins.

\section{Scheme 3}

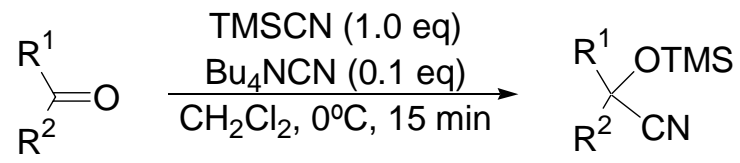

Table 2. O-TMS cyanosilylation of representative carbonyl compounds

\begin{tabular}{|c|c|c|c|}
\hline No & & Product $(\%)^{\mathrm{a}}$ & Diastereomeric ratio \\
\hline 1 & Benzaldehyde & 11a $(88)$ & --- \\
\hline $2^{d}$ & p-Methoxybenzaldehyde & $11 b(86)$ & --- \\
\hline $3^{\mathrm{e}}$ & 2-Furaldehyde & 11c $(86)$ & --- \\
\hline 4 & Cyclohexanone & 11d (89) & --- \\
\hline 5 & $\begin{array}{c}\text { Cyclopentanone } \\
\text { O }\end{array}$ & 11e (92) & --- \\
\hline 6 & & $11 f(95)$ & $3.3: 1^{\mathrm{b}}$ \\
\hline 7 & & $\operatorname{11g}(97)$ & $4.0: 1^{\mathrm{c}}$ \\
\hline
\end{tabular}

${ }^{a}$ Isolated yield of the diastereomeric O-TMS cyanohydrins.

${ }^{b}$ Determined by ${ }^{1} \mathrm{H}-\mathrm{NMR}$ on the purified mixture of diastereomeric O-TMS cyanohydrins.

${ }^{c}$ Determined by GC/MS on the purified mixture of diastereomeric O-TMS cyanohydrins.

${ }^{\mathrm{d}}$ The reaction was carried out in dry $\mathrm{Et}_{2} \mathrm{O}$.

${ }^{\mathrm{e}}$ The reaction was achieved at room temperature for $1 \mathrm{~h}$.

\section{Conclusions}

The formation of O-TMS cyanohydrins was possible for a variety of aldehydes and ketones using TMSCN as reagent and $\mathrm{Bu}_{4} \mathrm{NCN}$ as catalyst. The method is characterized by mild reaction conditions, short reaction times and good yields of the final O-TMS cyanohydrins. In those cases 
where the formation of stereoisomers is possible, an excess of one of the diastereomers was observed.

\section{Experimental Section}

Synthesis of 2,4-dibromo-3,5-dimethoxy-cyclohexa-2,4-diene-1-one-5-spirooxirane (5). Following the analogous synthetic route used by $\mathrm{K}$. Hinterding et $a{ }^{4}{ }^{4}$ to obtain the spiroepoxycyclohexadienone 1, 2,4,6-trimethoxybenzaldehyde was submitted to a four-step sequence:

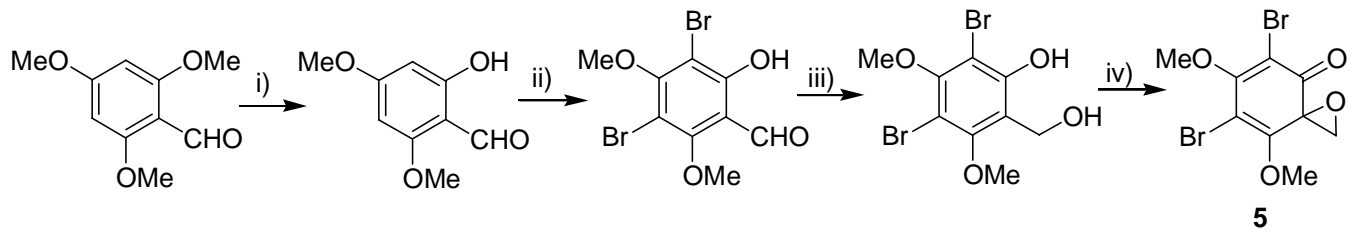

i) $\mathrm{BCl}_{3}, \mathrm{CH}_{2} \mathrm{Cl}_{2}, 4$ h., r. t., 91\%; ii) $\mathrm{Br}_{2} . \mathrm{HBrPyridine}$, Pyridine, 2 h., $50{ }^{\circ} \mathrm{C}, 76 \%$; iii) $\mathrm{NaBH}_{4}$, THF, 1 h., r. t., $80 \%$; iv) $\mathrm{NaIO}_{4}, \mathrm{HCl}, \mathrm{H}_{2} \mathrm{O}$, THF, r. t., $70 \%$.

Spectroscopic data for 5. ${ }^{1} \mathrm{H}-\mathrm{NMR}:\left(\mathrm{CDCl}_{3}, 200 \mathrm{MHz}\right) \delta 3.27\left(\mathrm{~d}, \mathrm{~J}=8.9 \mathrm{~Hz}, 1 \mathrm{H}, \mathrm{CH}_{2}-\mathrm{O}\right), 3.45$ $\left(\mathrm{d}, J=8.9 \mathrm{~Hz}, 1 \mathrm{H}, \mathrm{CH}_{2}-\mathrm{O}\right), 3.83\left(\mathrm{~s}, 3 \mathrm{H}, \mathrm{OCH}_{3}\right), 4.07$ (s, 3H, $\left.\mathrm{OCH}_{3}\right)$ ppm; ${ }^{13} \mathrm{C}-\mathrm{NMR}:\left(\mathrm{CDCl}_{3}, 50\right.$ $\mathrm{MHz}) \oint 57.47\left(\mathrm{O}-\mathrm{C}-\mathrm{CH}_{2}\right), 58.36\left(\mathrm{CH}_{2}-\mathrm{O}\right), 61.72\left(\mathrm{OCH}_{3}\right), 61.81\left(\mathrm{OCH}_{3}\right), 107.17(\mathrm{CBr}), 107.37$ $(\mathrm{CBr}), 159.11\left(\mathrm{C}-\mathrm{OCH}_{3}\right), 166.10\left(\mathrm{C}-\mathrm{OCH}_{3}\right), 185.48(\mathrm{C}=\mathrm{O}) \mathrm{ppm} ; \mathrm{MS}(70 \mathrm{eV}, \mathrm{EI}) \mathrm{m} / \mathrm{z}(\%)$ : 338/340/342 (52/94/44) [M+.], 323/325/327 (51/100/61) [M-15], 322/324/326 (51/57/35) [M-16], 241/243 (47/47), 59 (14).

\section{Typical procedure for the cyanosilylation of carbonyl compounds}

To a solution of the carbonyl compound $(0.367 \mathrm{mmol})$ in dry $\mathrm{CH}_{2} \mathrm{Cl}_{2}(0.5 \mathrm{~mL})$ was added, under Argon an at $0{ }^{\circ} \mathrm{C}$, TMSCN $(0.046 \mathrm{~mL}, 0.367 \mathrm{mmol})$ followed by a solution of the ammonium salt $(10 \mathrm{mg}, 0.037 \mathrm{mmol})$ in dry $\mathrm{CH}_{2} \mathrm{Cl}_{2}(0.5 \mathrm{~mL})$. The mixture was stirred at $0{ }^{\circ} \mathrm{C}$ for 15 minutes. A solution of $\mathrm{NaHCO}_{3}$ sat. $(3 \mathrm{~mL})$ was added and the mixture was extracted with $\mathrm{CH}_{2} \mathrm{Cl}_{2}$. Drying of the combined organic phases with $\mathrm{MgSO}_{4}$ was followed by evaporation of the solvent in vacuo. The products were purified by chromatography on silica gel (ethyl acetate/hexane) and characterized by ${ }^{1} \mathrm{H} \mathrm{NMR},{ }^{13} \mathrm{C} \mathrm{NMR}$ and mass spectrometry. For compounds 2+3, 8, 9 and $11 \mathrm{~g}$ the diastereomeric ratio was determined by GC/MS. Conditions: Capillary column $95 \%$ dimethyl $5 \%$ diphenylpolysiloxilane. Gradient of temperature $45^{\circ}-290^{\circ} \mathrm{C}$. Mass spectrometer, HP 5890 .

5,7-Dibromo-6-methoxy-4-trimethylsilanyloxy-1-oxa-spiro[2.5]octa-5,7-diene-4-carbonitrile (2) + (3). (a) ${ }^{1} \mathrm{H} \mathrm{NMR:}\left(\mathrm{CDCl}_{3}, 200 \mathrm{MHz}\right) \delta 0.41\left(\mathrm{~s}, 9 \mathrm{H}, 3 \mathrm{CH}_{3}-\mathrm{Si}\right), 2.91\left(\mathrm{~d}, J=5.0 \mathrm{~Hz}, 1 \mathrm{H}, \mathrm{CH}_{2}-\right.$ $\mathrm{O}), 3.44\left(\mathrm{~d}, J=5.0 \mathrm{~Hz}, 1 \mathrm{H}, \mathrm{CH}_{2} \mathrm{O}\right), 3.78\left(\mathrm{~s}, 3 \mathrm{H}, \mathrm{OCH}_{3}\right), 6.17$ (s, $\left.1 \mathrm{H}, \mathrm{CH}\right) \mathrm{ppm} ;{ }^{13} \mathrm{C}-\mathrm{NMR}$ : $\left(\mathrm{CDCl}_{3}, 75 \mathrm{MHz}\right) \delta\left(3 \mathrm{CH}_{3}-\mathrm{Si}\right), 50.16\left(\mathrm{CH}_{2}-\mathrm{CN}\right), 58.87\left(\mathrm{OCH}_{3}\right), 59.46\left(\mathrm{O}-\mathrm{C}-\mathrm{CH}_{2}\right), 73.70(\mathrm{O}-\mathrm{C}-$ $\mathrm{CN}), 109.10(\mathrm{CBr}), 114.68(\mathrm{CN}), 119.27(\mathrm{CBr}), 129.01(\mathrm{CH}), 148.87\left(\mathrm{C}-\mathrm{OCH}_{3}\right)$ ppm; MS (70 
eV, EI) m/z (\%): 407/409/411 (5/10/5) [M+'], 362/364/366 (11/22/11) [M-45], 352/354/356 (5/8/4) [M-55], 347/349/351 (3/7/4) [M-60], 337/339/341 (4/6/3) [M-70], 229/231 (18/18), 201/203 (7/7), 137/139 (5/5), 122 (5), 103 (6), 89 (8), 75(34), 74 (10), 73 (100), 59 (9), 45 (30), 44 (5), 43 (10).b) ${ }^{1} \mathrm{H}-\mathrm{NMR}:\left(\mathrm{CDCl}_{3}, 200 \mathrm{MHz}\right) \delta 0.28$ (s, 9H, 3CH $\left.\mathrm{CH}_{3}-\mathrm{Si}\right), 3.03(\mathrm{~d}, J=5.0 \mathrm{~Hz}, 1 \mathrm{H}$, $\mathrm{CH}_{2}-\mathrm{O}$ ), 3.42 (d, J=5.0 Hz, 1H, $\mathrm{CH}_{2}-\mathrm{O}$ ), 3.78 (s, $\left.3 \mathrm{H}, \mathrm{OCH}_{3}\right), 6.09$ (s, $\left.1 \mathrm{H}, \mathrm{CH}\right) \mathrm{ppm} ;{ }^{13} \mathrm{C}-\mathrm{NMR}$ : $\left(\mathrm{CDCl}_{3}, 75 \mathrm{MHz}\right) \delta\left(3 \mathrm{CH}_{3}-\mathrm{Si}\right), 51.75\left(\mathrm{CH}_{2}-\mathrm{CN}\right), 58.00\left(\mathrm{O}-\mathrm{C}_{-} \mathrm{CH}_{2}\right), 58.87\left(\mathrm{OCH}_{3}\right), 74.28(\mathrm{O}-\mathrm{C}-$ $\mathrm{CN}), 108.50(\mathrm{CBr}), 115.27(\mathrm{CN}), 118.93(\mathrm{CBr}), 128.72(\mathrm{CH}), 148.87\left(\mathrm{C}-\mathrm{OCH}_{3}\right) \mathrm{ppm}$; MS (70 eV, EI) m/z (\%): 407/409/411 (7/15/8) [M ${ }^{+}$], 363/365/367 (11/15/9) [M-44], 362/364/366 (39/78/40) [M-45], 352/354/356 (7/12/6) [M-55], 347/349/351 (6/13/8) [M-60], 229/231 (14/14), 201/203 (7/8), 137/139 (10/9), 103 (13), 89 (8), 75(32), 74 (11), 73 (100), 59 (14), 47 (11), 45 (32), 43 (12).

\section{5,7-Dibromo-6,8-dimethoxy-4-trimethylsilanyloxy-1-oxa-spiro[2.5]octa-5,7-diene-4-}

carbonitrile (8). (a) ${ }^{1} \mathrm{H}-\mathrm{NMR}:\left(\mathrm{CDCl}_{3}, 200 \mathrm{MHz}\right) \delta 0.14\left(\mathrm{~s}, 9 \mathrm{H}, 3 \mathrm{CH}_{3}-\mathrm{Si}\right), 3.09(\mathrm{~d}, 1 \mathrm{H}, J=5.4$ $\mathrm{Hz}, \mathrm{CH}_{2}-\mathrm{O}$ ), 3.22 (d, $\left.1 \mathrm{H}, J=5.4 \mathrm{~Hz}, \mathrm{CH}_{2}-\mathrm{O}\right), 3.61\left(\mathrm{~s}, 3 \mathrm{H}, \mathrm{OCH}_{3}\right), 3.73\left(\mathrm{~s}, 3 \mathrm{H}, \mathrm{OCH}_{3}\right)$ ppm. MS $\left(70 \mathrm{eV}\right.$, EI) $\mathrm{m} / \mathrm{z}$ (\%): 437/439/481 (20/40/21) $\left[\mathrm{M}^{+}\right.$] $]$392/394/396 (10/18/11) [M-45], 377/379/381 (10/21/12) [M-60], 358/360 (47/47) [M-Br], 335/337/339 (12/20/12) [M-104], 259/261 (49/50) [M-Br-TMSCN], 231/233 (22/21), 75 (46), 73 (100), 59 (27), 45 (25), 43 (26). (b) ${ }^{1} \mathrm{H}-\mathrm{NMR}$ : $\left(\mathrm{CDCl}_{3}, 200 \mathrm{MHz}\right) \delta 0.15\left(\mathrm{~s}, 9 \mathrm{H}, 3 \mathrm{CH}_{3}-\mathrm{Si}\right), 3.05\left(\mathrm{~d}, 1 \mathrm{H}, J=5.6 \mathrm{~Hz}, \mathrm{CH}_{2}-\mathrm{O}\right), 3.22$ (d, $\left.1 \mathrm{H}, J=5.6 \mathrm{~Hz}, \mathrm{CH}_{2}-\mathrm{O}\right), 3.61\left(\mathrm{~s}, 3 \mathrm{H}, \mathrm{OCH}_{3}\right), 3.70\left(\mathrm{~s}, 3 \mathrm{H}, \mathrm{OCH}_{3}\right)$ ppm. MS (70 eV, EI)

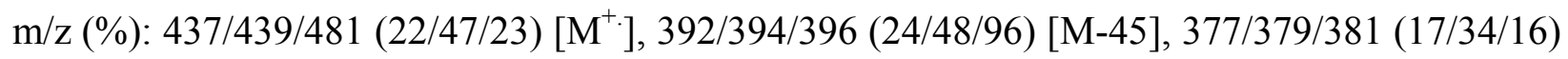
[M-60], 358/360 (34/34) [M-Br], 335/337/339 (16/32/15) [M-104], 259/261 (52/50) [M-BrTMSCN], 231/233 (21/19), 75 (28), 73 (100), 59 (28), 45 (32).

7-Bromo-4-trimethylsilanyloxy-1-oxa-spiro[2.5]octa-5,7-diene-4-carbonitrile (9). (a) ${ }^{1} \mathrm{H}$ NMR: $\left(\mathrm{CDCl}_{3}, 200 \mathrm{MHz}\right) \delta 0.20$ (s, 9H, 3CH $\left.-\mathrm{Si}\right), 2.83$ (d, $\left.1 \mathrm{H}, J=4.9 \mathrm{~Hz}, \mathrm{CH}_{2}-\mathrm{O}\right), 3.28$ (d, $1 \mathrm{H}$, $J=4.9 \mathrm{~Hz}, \mathrm{CH}_{2}-\mathrm{O}$ ), 5.70-5.90 (m, 2H, H-5, H-8), 6.21 (dd, $\left.1 \mathrm{H}, J=9.8 \mathrm{~Hz}, J=1.5 \mathrm{~Hz}, \mathrm{H}-6\right) \mathrm{ppm}$. MS (70 eV, EI) m/z (\%): 254/256 (18/19) [M-45], 244/246 (10/10) [M-55], 103 (17), 75 (20), 73 (100), 45 (66). (b) ${ }^{1} \mathrm{H}-\mathrm{NMR}:\left(\mathrm{CDCl}_{3}, 200 \mathrm{MHz}\right) \delta 0.19$ (s, 9H, 3CH $\mathrm{CH}_{3} \mathrm{Si}$ ), $2.89(\mathrm{~d}, 1 \mathrm{H}, J=5.0 \mathrm{~Hz}$, $\mathrm{CH}_{2}-\mathrm{O}$ ), 3.25 (d, $1 \mathrm{H}, J=4.9 \mathrm{~Hz}, \mathrm{CH}_{2}-\mathrm{O}$ ), 5.70-5.90 (m, 2H, H-5, H-8), 6.21 (dd, $1 \mathrm{H}, J=9.8 \mathrm{~Hz}$, $J=1,5 \mathrm{~Hz}, \mathrm{H}-6) \mathrm{ppm}$. MS (70 eV, EI) m/z (\%): 254/256 (79/81) [M-45], 103 (25), 75 (26), 73 (100), 45 (32).

6-Methoxy-4-trimethylsilanyloxy-1-oxa-spiro[2.5]octa-5,7-diene-4-carbonitrile (10). (a) ${ }^{1} \mathrm{H}$ NMR: $\left(\mathrm{CDCl}_{3}, 200 \mathrm{MHz}\right) \delta 0.02\left(\mathrm{~s}, 9 \mathrm{H}, 3 \mathrm{CH}_{3}-\mathrm{Si}\right), 2.94$ (d, $\left.1 \mathrm{H}, J=4.9 \mathrm{~Hz}, \mathrm{CH}_{2}-\mathrm{O}\right), 3.45$ (d, $1 \mathrm{H}$, $J=4.9 \mathrm{~Hz}, \mathrm{CH}_{2}-\mathrm{O}$ ), 4.87 (d, $\left.1 \mathrm{H}, J=2.5 \mathrm{~Hz}, \mathrm{H}-5\right), 5.55$ (d, $\left.1 \mathrm{H}, J=10.1 \mathrm{~Hz}, \mathrm{H}-8\right), 6.05$ (dd, $1 \mathrm{H}, J=$ $10.1 \mathrm{~Hz}, J=2.5 \mathrm{~Hz}, \mathrm{H}-7)$ ppm. b) ${ }^{1} \mathrm{H}-\mathrm{NMR}:\left(\mathrm{CDCl}_{3}, 200 \mathrm{MHz}\right) \delta 0.18\left(\mathrm{~s}, 9 \mathrm{H}, 3 \mathrm{CH}_{3}-\mathrm{Si}\right), 2.93(\mathrm{~d}$, $\left.1 \mathrm{H}, J=4.9 \mathrm{~Hz}, \mathrm{CH}_{2}-\mathrm{O}\right), 3.50$ (d, $1 \mathrm{H}, J=4.9 \mathrm{~Hz}, \mathrm{CH}_{2}-\mathrm{O}$ ), 4.81 (d, $\left.1 \mathrm{H}, J=2.5 \mathrm{~Hz}, \mathrm{H}-2\right), 5.55$ (d, $1 \mathrm{H}$, $J=10.1 \mathrm{~Hz}, \mathrm{H}-5$ ), 6.08 (dd, $1 \mathrm{H}, J=10.1 \mathrm{~Hz}, J=2.5 \mathrm{~Hz}, \mathrm{H}-4$ ) ppm.

2-Trimethylsilanyloxy-7-oxa-bicyclo[4.1.0] heptane-2-carbonitrile (11f). (a) ${ }^{1} \mathrm{H}-\mathrm{NMR}$ : $\left(\mathrm{CDCl}_{3}\right.$, $500 \mathrm{MHz}) \delta 0.30\left(\mathrm{~s}, 9 \mathrm{H}, 3 \mathrm{CH}_{3}-\mathrm{Si}\right), 1.40-2.10\left(\mathrm{~m}, 6 \mathrm{H}, 3 \mathrm{CH}_{2}\right), 3.38(\mathrm{t}, 1 \mathrm{H}, J=4.0 \mathrm{~Hz}, \mathrm{CH}-\mathrm{O}, \mathrm{H}-$ 6), $3.40(\mathrm{~d}, 1 \mathrm{H}, J=4.0 \mathrm{~Hz}, \mathrm{CH}-\mathrm{O}, \mathrm{H}-1) \mathrm{ppm} ;{ }^{13} \mathrm{C}-\mathrm{NMR}$ : $\left(\mathrm{CDCl}_{3}, 50 \mathrm{MHz}\right) \delta 1.18\left(3 \mathrm{CH}_{3}-\mathrm{Si}\right)$, $18.82\left(\mathrm{CH}_{2}\right), 21.16\left(\mathrm{CH}_{2}\right), 32.03\left(\mathrm{CH}_{2}, \mathrm{C}-3\right), 55.09(\mathrm{CH}), 56.71(\mathrm{CH}), 70.65(\mathrm{C}), 119.72(\mathrm{CN})$ 
ppm. b) ${ }^{1} \mathrm{H}-\mathrm{NMR}:\left(\mathrm{CDCl}_{3}, 500 \mathrm{MHz}\right) \delta 0.32\left(\mathrm{~s}, 9 \mathrm{H}, 3 \mathrm{CH}_{3}-\mathrm{Si}\right), 1.40-2.10\left(\mathrm{~m}, 6 \mathrm{H}, 3 \mathrm{CH}_{2}\right), 3.20$ (d, $1 \mathrm{H}, J=3.5 \mathrm{~Hz}, \mathrm{CH}-\mathrm{O}, \mathrm{H}-1), 3.34$ (t, $1 \mathrm{H}, J=3.5 \mathrm{~Hz}, \mathrm{CH}-\mathrm{O}, \mathrm{H}-6) \mathrm{ppm}$.

2-Trimethylsilanyloxy-6-oxa-bicyclo[3.1.0]hexane-2-carbonitrile (11g). (a) ${ }^{1} \mathrm{H}-\mathrm{NMR}$ : $\left(\mathrm{CDCl}_{3}\right.$, $500 \mathrm{MHz}) \delta 0.29\left(\mathrm{~s}, 9 \mathrm{H}, 3 \mathrm{CH}_{3}-\mathrm{Si}\right), 1.75(\mathrm{dd}, 1 \mathrm{H}, J=11.7 \mathrm{~Hz}, J=8.5 \mathrm{~Hz}, \mathrm{H}-4), 1.83$ (dd, $1 \mathrm{H}$, $J=11.7 \mathrm{~Hz}, J=9.0 \mathrm{~Hz}, \mathrm{H}-3$ ), 2.12 (dd, $1 \mathrm{H}, J=13.3 \mathrm{~Hz}, J=9.0 \mathrm{~Hz}, \mathrm{H}-3), 2.21$ (dd, 1H, $J=13.3 \mathrm{~Hz}$, $J=8.5 \mathrm{~Hz}, \mathrm{H}-3), 3.56(\mathrm{~d}, 1 \mathrm{H}, J=2.6 \mathrm{~Hz}, \mathrm{H}-5), 3.66$ (d, 1H, $J=2.6 \mathrm{~Hz}, \mathrm{H}-1) \mathrm{ppm} ;{ }^{13} \mathrm{C}-\mathrm{NMR}$ : $\left(\mathrm{CDCl}_{3}, 50 \mathrm{MHz}\right) \delta .\left(3 \mathrm{CH}_{3}-\mathrm{Si}\right), 24.91\left(\mathrm{CH}_{2}, \mathrm{C}-4\right), 32.62\left(\mathrm{CH}_{2}, \mathrm{C}-3\right), 55.17(\mathrm{CH}), 59.64(\mathrm{CH})$, 74.91 (C), $119.61(\mathrm{CN}) \mathrm{ppm}$; MS (70 eV, EI) m/z (\%): 182 (49) [M-CH $], 155$ (34) $\left[{\mathrm{M}-\mathrm{CH}_{3}-}^{-}\right.$ HCN], 127 (81), 84 (28), 81 (33), 75 (55), 73 (100), 45 (44), 43 (22), 41 (75). b) ${ }^{1} \mathrm{H}-\mathrm{NMR}:$ $\left(\mathrm{DMSO}_{-} \mathrm{d}_{6}, 500 \mathrm{MHz}\right) \delta 0.22\left(\mathrm{~s}, 9 \mathrm{H}, 3 \mathrm{CH}_{3}-\mathrm{Si}\right), 1.50-2.10\left(\mathrm{~m}, 4 \mathrm{H}, 2 \mathrm{CH}_{2}\right), 3.73(\mathrm{~d}, 1 \mathrm{H}, J=2.5 \mathrm{~Hz}$, H-5), 3.82 (d, 1H, J=2.5 Hz, H-1) ppm; MS (70 eV, EI) m/z (\%): 182 (21) [M-CH 3 , 155 (69) [M-CH $3-\mathrm{HCN}$ ], 126 (12), 113 (14), 101 (13), 84 (16), 81 (35), 75 (28), 73 (100), 47 (13), 45 (35), 43 (16).

\section{Acknowledgements}

Thanks are given to the DGI/MCyT of Spain for financial support (project number BQU-20000653).

\section{References}

1. For a recent review, see: Gregory, R. J. H. Chem. Rev.1999, 99, 3649

2. See ref. 1 and: (a) Saravan, P.; Anand, R. V.; Singh, V. K. Tetrahedron Lett. 1998, 39, 3823. (b) Somathan, R.; Rivero, I. A.; Gama, A.; Ochoa, A.; Aguirre, G. Synth. Commun. 1998, 28, 2043. (c) Kantam, M. L.; Sreekanth, P.; Santhi, P. L. Green Chem. 2000, 2, 47. (d) Wang, Z. G.; Fetterly, B.; Verkade, J. G. J. J. Organomet. Chem. 2002, 646, 161. (e) Yadav, J. S.; Reddy, M. S.; Prasad, A. R. Tetrahedron Lett. 2002, 43, 9703. (f) Bandini, M.; Cozzi, P. G.; Garelli, A.; Melchiorre, P.; Umani-Ronchi, A. Eur. J. Org. Chem. 2002, 3243. (g) Córdoba, R.; Plumet, J. Tetrahedron Lett. 2003, 44, 6157.

3. Spiroepoxycyclohexadienones are useful synthetic intermediates mainly in the context of their reactivity as dienes in Diels-Alder reactions. For some selected accounts, see: (a) Singh, V. Acc. Chem. Res. 1999, 32, 324. (b) Bonnarme, V.; Bachmann, Ch.; Cousson, A.; Mondon, M.; Gesson, J. P. Tetrahedron 1999, 55, 433. (c) Bonnarme, V.; Mondon, M.; Cousson, A.; Gesson, J. P. Chem. Comm. 1999, 1143. (d) Quideau, S.; Poységu, L. Org. Prep. Proc. Int. 1999, 31, 617.

4. Hinterding, K.; Knebel, A.; Herrlich, P.; Waldmann, H. Bioorg. Med. Chem. 1998, 6, 1153.

5. Evans, D. A.; Truesdale, L. K. Tetrahedron Lett. 1973, 14, 4929. 